OPEN ACCESS

Edited by:

Carlo Baldari,

Foro Italico University of Rome, Italy

Reviewed by:

David Rodríguez-Rosell, Universidad Pablo de Olavide, Spain Argyris G. Toubekis,

National and Kapodistrian University of Athens, Greece

*Correspondence: Daniel A. Marinho marinho.d@gmail.com

Specialty section: This article was submitted to

Exercise Physiology, a section of the journa

Frontiers in Physiology

Received: 02 August 2019 Accepted: 09 December 2019 Published: 09 January 2020

Citation:

Cerqueira É, Marinho DA, Neiva HP and Lourenço O (2020) Inflammatory Effects of High and Moderate Intensity Exercise-A Systematic Review. Front. Physiol. 10:1550. doi: 10.3389/fphys.2019.01550

\section{Inflammatory Effects of High and Moderate Intensity Exercise-A Systematic Review}

\author{
Érica Cerqueira ${ }^{1}$, Daniel A. Marinho ${ }^{2,3 *}$, Henrique P. Neiva ${ }^{2,3}$ and Olga Lourenço ${ }^{1,4}$ \\ ${ }^{1}$ FCS - UBI, Faculty of Health Sciences, University of Beira Interior, Covilha, Portugal, ${ }^{2}$ Department of Sport Sciences, \\ University of Beira Interior, Covilha, Portugal, ${ }^{3}$ Research Center in Sport Sciences, Health Sciences and Human Development \\ (CIDESD), Covilha, Portugal, ${ }^{4} \mathrm{CICS}-\mathrm{UBI}$, Health Sciences Research Centre, University of Beira Interior, Covilha, Portugal
}

Background: Exercise leads to a robust inflammatory response mainly characterized by the mobilization of leukocytes and an increase in circulating inflammatory mediators produced by immune cells and directly from the active muscle tissue. Both positive and negative effects on immune function and susceptibility to minor illness have been observed following different training protocols. While engaging in moderate activity may enhance immune function above sedentary levels, excessive amounts of prolonged, high-intensity exercise may impair immune function. Thus, the aim of the present review was to clarify the inflammatory effects in response to different exercise intensities.

Methods: Search was performed on PubMed and was completed on July 31st, 2017. The studies were eligible if they met the predefined inclusion criteria: a) observational or interventional studies, b) conducted in healthy adults (18-65 years), c) written in Portuguese, English or Spanish, d) including moderate and/or intense exercise. Eighteen articles were included. The specific components that were examined included circulating blood levels of cytokines, leukocytes, creatine kinase (CK) and C-reactive protein (CRP). The methodological quality of the included studies was assessed.

Results: Most of the intervention studies showed changes in the assessed biomarkers, although these changes were not consistent. White blood cells (WBC) had an increase immediately after intensive exercise ( $>64 \% \mathrm{VO}_{2 \max }$ ), without alteration after moderate exercise (46-64\% $\mathrm{VO}_{2 \max }$ ). The results suggested an elevation of the pro-inflammatory cytokines, namely IL-6, followed by an elevation of IL-10 that were more evident after intense exercise bouts. CRP increased both after intense and moderate exercise, with peak increases up to $28 \mathrm{~h}$. CK increased only after intensive and long exercising.

Conclusion: In summary, intense long exercise can lead, in general, to higher levels of inflammatory mediators, and thus might increase the risk of injury and chronic inflammation. In contrast, moderate exercise or vigorous exercise with appropriate resting periods can achieve maximum benefit.

Keywords: inflammation, exercise, high intensity, moderate intensity, cytokines 


\section{INTRODUCTION}

Inflammation is characterized by a cascade of cellular and molecular events leading to an increase in body temperature, capillary dilatation, and production of blood-borne soluble components (Allen et al., 2015; Gonzalo-Calvo et al., 2015). These responses, which can be induced by stressors and are vital for host defense and natural tissue homeostasis, initiate the elimination of noxious compounds and damaged tissue (Moldoveanu et al., 2001; Allen et al., 2015).

Exercise works as a stressor during and after its execution and it is able to cause inflammation (Silveira et al., 2016). Interestingly, however, regular physical exercise training may be considered a long-lasting anti-inflammatory therapy, after the acute inflammatory actions are resolved (Gleeson et al., 2011; Allen et al., 2015). Moreover, pro-inflammatory processes that occur after exercise, such as increases in the expression of proinflammatory cytokines, may be vital for the long-term adaptive responses to exercise training. Inflammation is essential in order to repair processes to occur, like those resulting from exercise and training (Oishi and Manabe, 2018). Consequently, exerciseinduced changes in inflammation can be divided into acute effects (changes during and immediately following a bout of exercise) and long-term effects (changes in resting or basal levels, when the acute exercise-induced effects have been washed out) (Roca-rodríguez et al., 2015; Pedersen, 2017).

Some authors have suggested that acute exercise bouts initiate a complex cascade of inflammatory events, which depend on the type, intensity, duration and familiarity of the exercise, as well as the age and clinical condition of the participants (Moldoveanu et al., 2001; Allen et al., 2015; Bigley and Simpson, 2015; Silveira et al., 2016; Hennigar et al., 2017; Peake et al., 2017). Measurable immune parameters affected by exercise comprise changes in peripheral blood cell numbers, granulocyte activity, NK cell cytotoxic activity, lymphocyte proliferation, and cytokine levels in plasma, among others (Moldoveanu et al., 2001; Petersen and Pedersen, 2005; Timmons and Cieslak, 2008; Gleeson et al., 2011; Allen et al., 2015; Bigley and Simpson, 2015; Lightfoot and Cooper, 2016; Hennigar et al., 2017; Peake et al., 2017; Shaw et al., 2017).

Cytokines are soluble proteins or glycoproteins, produced and segregated during inflammation, that mediate the communication between immune and non-immune cells and regulate biological processes (Chen et al., 2018). The production of cytokines can be upregulated rapidly in response to inflammatory stimuli, and this response can be transient or prolonged (Allen et al., 2015). The pro-inflammatory cytokines (TNF- $\alpha$, IL-1 $\beta$, and IL-6) are released after physical activity of

\footnotetext{
Abbreviations: CK, creatine kinase; CONSORT, Consolidated Standards of Reporting Trials; CRP, C-reactive protein; $\mathrm{HR}_{\max }$, maximal heart rate; $\mathrm{HRR}_{\max }$, Maximal heart rate reserve; IL, Interleukin; MEDLINE, MEDical Literature Analysis and Retrieval System; MET, Metabolic equivalent; NK, Natural killer; PBMC, Peripheral Blood Mononuclear Cells; PICO, Population, Intervention, Comparison and Outcome; PRISMA, Preferred Reporting Items for Systematic reviews and Meta-Analysis; RM, Repetition maximum; STROBE, Strengthening the reporting of observational studies in epidemiology; TNF- $\alpha$, Tumor necrosis factor alpha; $\mathrm{VO}_{2 \max }$, Maximal oxygen consumption; WBC, White Blood Cells.
}

sufficient intensity, followed by the release of anti-inflammatory or regulatory cytokines (IL-4, IL-10, IL-1RA, and IL-13) that attenuate that response (Moldoveanu et al., 2001).

Several proteins are affected in response to inflammatory processes, the majority showing increased levels shortly after an inflammatory reaction (Fedewa et al., 2016). Those proteins whose concentration increases are referred to as positive acutephase proteins. CRP is a hepatic acute-phase protein, a marker of systemic inflammation and is associated with cardiovascular risk (Allen et al., 2015). Moreover, its levels have been correlated with frailty, morbidity, and mortality (Allen et al., 2015). CRP has lower levels in people who do moderate exercise compared to inactive people (Allen et al., 2015; Fedewa et al., 2016). Creatine kinase $(\mathrm{CK})$ is a protein involved in muscle metabolism, and its concentration is generally considered a physical stress marker (Moghadam-Kia et al., 2016). Leakage of CK into the plasma is accepted as a semi-quantitative indicator of muscle fiber damage (Marqués-Jiménez et al., 2016). CK levels have a significant variation with sex and race and also with exercise type: eccentric exercise causing more muscle damage than concentric contractions of the same vigor (Baumert et al., 2016; MoghadamKia et al., 2016).

Overall, it seems evident that there are immune changes after exercise, especially with increased intensity. Moreover, there is a belief that these changes differ markedly after heavy exercitation from those following moderate exercise. Therefore, this systematic review aimed to synthesize and analyze the moderate and intense physical activity in healthy active adults, to explore the associated inflammation markers, and to provide quantitative estimates on the change of these markers.

\section{METHODS}

\section{Search Strategy}

A comprehensive search in the MEDLINE (PubMed) database was conducted (NCBI) ${ }^{1}$ The main target was to find studies that described immunologic changes in response to moderate and/or intense/vigorous exercise. The search expression ("Inflammation"[Mesh]) AND ("Exercise"[Mesh]) was used in all fields (Table 1). No limitation was made in publication date or duration of the study. Literature published from the inception of the database up to 31 July 2017 was included.

\section{Eligibility Criteria}

After the initial search, duplicates and studies not relevant for this analysis were excluded and the remaining studies' abstracts were examined by two independent reviewers. Doubts regarding the inclusion or exclusion of studies were resolved by discussion between the two independent researchers. After this first selection, both researchers read through the articles to decide whether they were eligible using criteria defined with the PICO (Population, Intervention, Comparison, and Outcome) criteria (Table 1; Methley et al., 2014). Further studies were considered for inclusion after verifying the references of the original studies. As no other databases were searched, manual

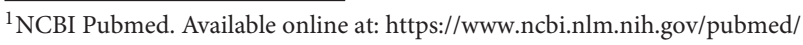


TABLE 1 | Search strategy and inclusion/exclusion criteria based on PICO.

\begin{tabular}{|c|c|c|c|c|}
\hline Databases & $\begin{array}{l}\text { Search } \\
\text { terms }\end{array}$ & PICO & Inclusion criteria & $\begin{array}{l}\text { Exclusion } \\
\text { criteria }\end{array}$ \\
\hline \multirow[t]{5}{*}{ PubMed } & $\begin{array}{l}\text { Inflammation } \\
\text { Exercise }\end{array}$ & Population & $\begin{array}{l}\text { Healthy adults } \\
\text { (18-65 years) }\end{array}$ & $\begin{array}{l}\text { Sedentary adults; } \\
\text { adults with disease }\end{array}$ \\
\hline & & Intervention & Moderate exercise & $\begin{array}{l}\text { No exercise } \\
\text { intensity definition }\end{array}$ \\
\hline & & & Intense exercise & \\
\hline & & Comparison & $\begin{array}{l}\text { Intense with moderate } \\
\text { exercise }\end{array}$ & \\
\hline & & Outcome & $\begin{array}{l}\text { Alterations in PBMC } \\
\text { (WBC, lymphocytes, } \\
\text { NK cells, or NK } \\
\text { cytolytic activity), } \\
\text { Cytokines (IL-6, IL-8, } \\
\text { IL-1 } \beta \text {, TNF- } \alpha \text {, or IL-10), } \\
\text { CRP, or CK }\end{array}$ & $\begin{array}{l}\text { No results on } \\
\text { inflammatory } \\
\text { markers }\end{array}$ \\
\hline
\end{tabular}

searches were performed in the reference lists of all included studies and relevant review studies.

\section{Risk of Bias Assessment}

Scientific quality of the studies was assessed independently by two reviewers using the STROBE scale for cross-sectional studies and the CONSORT scale for clinical trials (von Elm et al., 2008; Schulz and Atlman, 2010). If assessment outcomes were conflicting, a consensus-based final score was attributed.

\section{Data Extraction and Analysis}

This systematic literature review about inflammatory effects following high and moderate intensity exercise was registered in PROSPERO (CRD 42018085835) and was performed according to the recommendations established by the Preferred Reporting Items for Systematic reviews and Meta-Analysis (PRISMA) statement guidelines (Urrútia and Bonfill, 2010).

The main participants' characteristics and the main study outcomes were identified. Data regarding type, intensity and duration of physical exercise and exercise-induced changes in inflammation markers were identified and appraised. Moderate exercise was defined by: Borg scale between 12 and 13 or $\%$ of maximal heart rate $\left(\mathrm{HR}_{\max }\right)$, between 64 and $76 \%$ or $\%$ maximal heart rate reserve $\left(\mathrm{HR}_{\max }\right)$, between 40 and $60 \%$ or \% maximal oxygen consumption $\left(\mathrm{VO}_{2 \max }\right)$, between 46 and $64 \%$ or metabolic equivalent (MET), between 3 and 6 or $\%$ repetition maximum (RM), between 50 and $70 \%$ or MET by age between 4.8 and 7.2 (young: 20-39 years) and 4.06.0 (middle age: 40-64 years). Intense exercise was defined by: Borg scale $>13$ or $\mathrm{HR}_{\max }>76 \%$ or $\mathrm{HR}_{\max }>60 \%$ or $\mathrm{VO}_{2 \max }>64 \%$ or MET $>6.0$ or $\mathrm{RM}>70 \%$ or MET by age $>7.2$ (young: 20-39 years) and $>6.0$ (middle age: $40-$ 64 years) (Pescatello et al., 2013). The effects of the exercise intensities on inflammatory markers were evaluated in blood samples collected before and after the exercise bouts and relative increases related to the baseline levels were determined (number of times). Moreover, this analysis was calculated using Cohen's $d$, where the mean post-value was subtracted from the mean pre-value and divided by the standard deviation. This method allowed to determine the magnitude of differences obtained with the experimental treatment. The magnitude of the effect was classified as small $(d=0.2)$, intermediate $(d=0.5)$, or large $(d=0.8)$ (Cohen, 1988).

\section{RESULTS}

\section{Characteristics of the Interventional Studies}

A total of 1,374 records were identified through database searching and 7 additional records were identified through other sources. After removal of duplicates, 1,380 articles were considered for abstract reading. Of the 41 selected articles, only 39 were available as full-text and assessed for eligibility. After full-text reading, 18 studies were included for quality synthesis (Figure 1). Most of the included studies showed intermediate to good quality (Tables S1, S2).

The eighteen (18) included studies collected data from 255 healthy subjects. These participants performed different kinds of exercise interventions (running, cycling, resistance training, and kayaking) in high and/or moderate exercise intensities. The age of the individuals ranged from 18 to 53 years $(35.9 \pm 17.0)$. Some of the results were from studies of mixed gender (Abbasi et al., 2013; Stelzer et al., 2015) and the remainder from male volunteers only (Brenner et al., 1999; Mucci et al., 1999; Ostrowski et al., 1999; Connolly et al., 2004; Degerstrøm and Østerud, 2006; Fatouros et al., 2006; Spiropoulos et al., 2010; Bernecker et al., 2011; Nieman et al., 2012; Bonsignore et al., 2013; Draganidis et al., 2013; Marklund et al., 2013; Azizbeigi et al., 2015; GonzaloCalvo et al., 2015; Ulven et al., 2015; Wadley et al., 2015). In the studies where the volunteers did more than one bout of exercise in different intensities, the resting period was highly variable: 1 month (Gonzalo-Calvo et al., 2015), 3 weeks (Draganidis et al., 2013), 2 weeks (Brenner et al., 1999), 1 week (Mucci et al., 1999; Ulven et al., 2015; Wadley et al., 2015), or 5 days (Fatouros et al., 2006). The characteristics of the included studies are summarized in Table 2.

The comparison in terms of inflammatory biomarkers between moderate and intense exercise is presented in Table 3. For most studies, blood samples were taken minutes after exercise (immediately, 10 or $15 \mathrm{~min}$ ). However, some markers were evaluated at other time points: IL- 10 (Wadley et al., 2015), WBC, and lymphocytes (Abbasi et al., 2013) were evaluated also $30 \mathrm{~min}$ after exercise; WBC, IL-6, and TNF- $\alpha$ (Bernecker et al., 2011) were evaluated also $1 \mathrm{~h}$ after exercise; IL-10 and TNF- $\alpha$ (Brenner et al., 1999) were evaluated also $3 \mathrm{~h}$ after exercise and CK (Brenner et al., 1999) was evaluated also 3 days after exercise; CRP and CK (Fatouros et al., 2006) were evaluated also 4 days after exercise. Table 3 considers measurements collected up to $15 \mathrm{~min}$ after exercise. The results of IL-6 and CRP from Spiropoulos et al. (2010) were not considered because they showed increases of 10,470 and 6,000 times, respectively, which makes them noncomparable with the other studies. The same occurs with the study by Marklund et al. (2013) in which the baseline value of CRP wasn't detectable. 


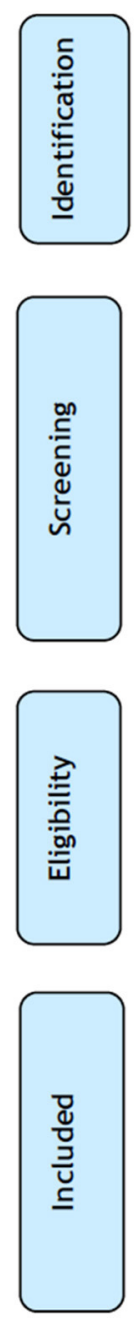

Records identified through database searching $(n=1374)$

\section{Additional records of reference} lists of all included studies

$(\mathrm{n}=7)$

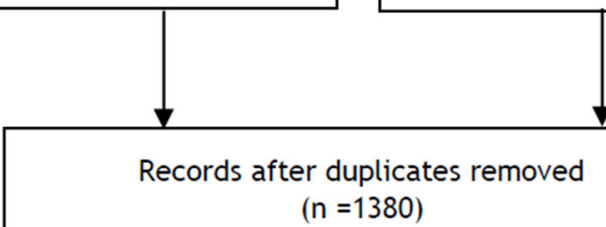

$(n=1380)$

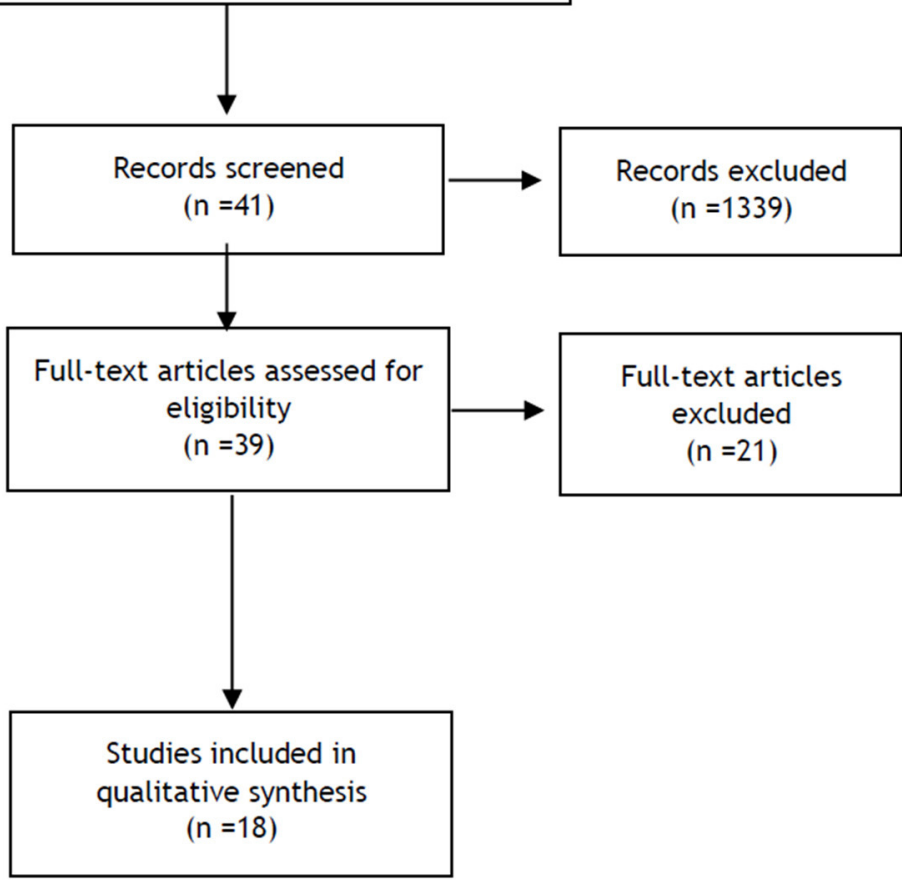

FIGURE 1 | PRISMA (preferred reporting items for systematic reviews and meta-analyses) study flow diagram.

\section{Effects of Exercise on Cytokine Secretion}

Fifteen studies evaluated the effects of exercise on cytokine concentration in blood (IL-6, IL-8, IL-1 $\beta$, IL-10, and TNF- $\alpha$ ). Our review supports, in general, the idea that exercise can stimulate both pro- and anti-inflammatory responses. These increases were transitory, with the values returning to baseline sometime from 5 to $24 \mathrm{~h}$ after exercise.

IL-6 was the cytokine more often evaluated (13 studies) corresponding to 4 moderate and 12 intense exercise bouts. There were increases in IL- 6 after exercise ranging from 1.33 to 4.20 times in moderate and from 1.59 to 26.79 in high-intensity exercises, immediately after exercise. In 6 studies there was no increase ( 2 moderate and 4 intense exercises) (Brenner et al., 1999; Degerstrøm and Østerud, 2006; Bernecker et al., 2011; Azizbeigi et al., 2015).

IL-8 increased after moderate exercise and after intense exercise ranging from 1.37 to 2.77 times (Mucci et al., 1999; Degerstrøm and Østerud, 2006; Nieman et al., 2012; Marklund et al., 2013; Gonzalo-Calvo et al., 2015). IL-10 increased after intense exercise ranging from 1.57 to 32.99 times in 4 studies (Ostrowski et al., 1999; Nieman et al., 2012; Gonzalo-Calvo et al., 2015; Ulven et al., 2015). In three studies there was no increase, one of these referred to intense and moderate exercise, one to moderate and another to intense exercise only.

Gonzalo-Calvo et al. (2015) evaluated the effect of moderate and intense exercise on circulating IL-8, IL-6, and IL-10, and observed an increase in all the evaluated cytokines. This rise was maintained for more than 1 day and then returned to baseline. Wadley et al. (2015) evaluated the cytokine profile after moderate and intense cycling exercise. Their results show an increase in IL- 6 at 30 min after exercise independently of intensity. In the same study, IL-10 increases $15 \mathrm{~min}$ after intense exercise, without alteration in moderate exercise (Wadley et al., 2015). Mucci et al. (1999) evaluated cycling intense and moderate exercises showing a transient increase in IL-8 in both intensities with a return to baseline values after $5 \mathrm{~min}$. Brenner et al. (1999) evaluated 
TABLE 2 | Studies characteristics.

\begin{tabular}{|c|c|c|c|c|c|c|}
\hline References & Study type & $\begin{array}{l}\text { Intensity and description } \\
\text { of exercise }\end{array}$ & Subjects & $\begin{array}{l}\text { Inflammatory } \\
\text { markers }\end{array}$ & Measure points & Findings \\
\hline \multirow[t]{2}{*}{$\begin{array}{l}\text { Gonzalo-Calvo et al. } \\
\text { (2015) }\end{array}$} & Cross-sectional & $\begin{array}{l}\text { Intense: } 10 \mathrm{~km} \text {-race } \\
\left(89.12 \% \mathrm{VO}_{2 \max }\right)\end{array}$ & $\begin{array}{l}9 \mathrm{M} \\
\text { Amateur runners } \\
\text { Training experience: } \\
6.6 \pm 5.0 \mathrm{yr} \text { and } \\
69.7 \pm 5.0 \mathrm{~km} / \mathrm{wk}\end{array}$ & $\begin{array}{l}\text { PBMC: WBC, } \\
\text { lymphocytes } \\
\text { Cytokines: IL-6, } \\
\text { IL-8, and IL-10 }\end{array}$ & $\begin{array}{l}1.5 \mathrm{~h} \text { before exer, } \\
10 \text { min, } 1 \text { day and } \\
3 \text { days after }\end{array}$ & $\begin{array}{l}\uparrow W B C, \text { Lymphocytes and NK cells } \\
10 \text { min after exer } \\
\uparrow C K \text { from } 10 \text { min to } 1 \text { day after exer } \\
\leftrightarrow \mid \mathrm{L}-8, \text { IL-6, IL-10, and CRP }\end{array}$ \\
\hline & & $\begin{array}{l}\text { Intense: Marathon }(68.70 \% \\
\left.\mathrm{VO}_{2 \max }\right)\end{array}$ & & & & $\begin{array}{l}\uparrow W B C, N K \text { cells, IL-6, IL-8, IL-10 } \\
\text { and CRP } 10 \text { min after exer } \\
\uparrow C K \text { and CRP from } 10 \text { min to } 1 \text { day } \\
\text { and } \downarrow \text { after that } \\
\leftrightarrow \text { Lymphocytes }\end{array}$ \\
\hline \multirow[t]{2}{*}{ Wadley et al. (2015) } & Cross-sectional & $\begin{array}{l}\text { Intense: LV-HIIE ( } 90 \% \\
\left.\mathrm{VO}_{2 \max }\right) \text { and high ( } 80 \% \\
\left.\mathrm{VO}_{2 \max }\right) \\
10 \times 1 \text { min cycling of } \\
\mathrm{LV}-\mathrm{HIIE} \text { with } 1 \text { min interval } \\
20 \text { min cycling of } \\
\text { high exercise }\end{array}$ & $\begin{array}{l}10 \mathrm{M} \\
\text { Untrained }\end{array}$ & $\begin{array}{l}\text { PBMC: } \\
\text { Lymphocytes } \\
\text { Cytokines: IL-6 } \\
\text { and IL-10 }\end{array}$ & $\begin{array}{l}\text { Before, at the end } \\
\text { and } 30 \text { min after } \\
\text { exer }\end{array}$ & $\begin{array}{l}\uparrow \text { Lymphocytes at the end, returns } \\
\text { to baseline in } 30 \text { min } \\
\uparrow I L-1030 \text { min at the end } \\
\uparrow I L-6 \text { at the end and } 30 \text { min } \\
\text { after exer }\end{array}$ \\
\hline & & $\begin{array}{l}\text { Moderate: cycled for } 27 \mathrm{~min} \\
\left(60 \% \mathrm{VO}_{2 \max }\right)\end{array}$ & & & & $\begin{array}{l}\uparrow \text { Lymphocytes at the end and } \\
\text { returns to baseline in } 30 \text { min } \\
\uparrow I L-6 \text { at the end and } 30 \text { min after } \\
\text { exer } \\
\leftrightarrow I L-10\end{array}$ \\
\hline Ulven et al. (2015) & Cross-sectional & $\begin{array}{l}\text { Intense: Cycled for } 1 \mathrm{~h} \\
\text { repeated twice } \\
\left(70 \% \mathrm{VO}_{2 \max }, \% \mathrm{HR}_{\max } \cong\right. \\
87.8 \% \text {, and Borg scale } \\
\cong 15.4)\end{array}$ & $\begin{array}{l}10 \mathrm{M} \\
\text { Very good } \\
\text { physical fitness }\end{array}$ & $\begin{array}{l}\text { Cytokines: IL-6, } \\
\text { IL-10, and TNF- } \alpha\end{array}$ & $\begin{array}{l}\text { Before and at the } \\
\text { end of cycle test }\end{array}$ & $\begin{array}{l}\text { 个IL-6, IL-10, and TNF- } \alpha \text { at the end } \\
\text { of exer }\end{array}$ \\
\hline Stelzer et al. (2015) & Cross-sectional & $\begin{array}{l}\text { Intense: Cycling race } \\
\left(98.68 \% \mathrm{HR}_{\max }\right) \\
8 \mathrm{~h} \text { of competition and } \\
8 \mathrm{~h} \text { of rest during } 4 \text { days }\end{array}$ & $\begin{array}{l}7 \text { (3 F: } 4 \mathrm{M}) \\
\text { Moderately trained } \\
\text { amateur athletes } \\
\text { Training experience } \\
7.5 \pm 3.9 \mathrm{~h} / \mathrm{wk}\end{array}$ & $\begin{array}{l}\text { PBMC: WBC and } \\
\text { lymphocytes } \\
\text { Cytokines: IL-6 } \\
\text { CK }\end{array}$ & $\begin{array}{l}2 \text { days pre-race } \\
\text { and } 15 \mathrm{~min} \\
\text { post-race }\end{array}$ & $\begin{array}{l}\uparrow W B C \text {, lymphocytes, IL-6, and CK } \\
\text { post-race }\end{array}$ \\
\hline Abbasi et al. (2013) & Cross-sectional & $\begin{array}{l}\text { Intense: } \mathrm{HM} \text { in competition } \\
\text { conditions } \\
\mathrm{N} \cong 13.26 \mathrm{~km} / \mathrm{h} \text { for men } \\
\text { and } 11.11 \mathrm{~km} / \mathrm{h} \text { for women) } \\
\text { Timing: } 95.5 \pm 8 \mathrm{~min} \text { for } \\
\text { men and } 114 \pm 12 \mathrm{~min} \\
\text { for women }\end{array}$ & $\begin{array}{l}16 \text { (8 F: } 8 \mathrm{M}) \\
\text { Well-trained athletes } \\
\text { Training experience: } \\
\text { endurance training } \\
\text { for at least } 2 \mathrm{yr} ;\end{array}$ & $\begin{array}{l}\text { PBMC: WBC and } \\
\text { lymphocytes; }\end{array}$ & $\begin{array}{l}\text { Before, } 30 \mathrm{~min}, \\
3 \mathrm{~h} \text { and } 24 \mathrm{~h} \text { after } \\
\text { exer; }\end{array}$ & $\begin{array}{l}\uparrow W B C \text { at } 30 \mathrm{~min} \text { and } 3 \mathrm{~h} \text { after exer } \\
\downarrow \text { Lymphocytes at } 30 \mathrm{~min} \text { and } 3 \mathrm{~h} \\
\text { after exer }\end{array}$ \\
\hline
\end{tabular}


TABLE 2 | Continued

\begin{tabular}{|c|c|c|c|c|c|c|}
\hline References & Study type & $\begin{array}{l}\text { Intensity and description } \\
\text { of exercise }\end{array}$ & Subjects & $\begin{array}{l}\text { Inflammatory } \\
\text { markers }\end{array}$ & Measure points & Findings \\
\hline \multirow[t]{2}{*}{$\begin{array}{l}\text { Draganidis et al. } \\
\text { (2013) }\end{array}$} & Cross-sectional & $\begin{array}{l}\text { Intense: Resistance training: } \\
\text { squat, seated leg extension, } \\
\text { horizontal leg curls, barbell } \\
\text { side lunges, and calf raises; } \\
\text { (85-90\% 1 RM) } \\
4 \text { sets, } 4-6 \text { repetitions } \\
\text { per set with } 3 \text { min rest } \\
\text { Training during around } \\
40-45 \text { min following of } \\
10 \text { min warm-up }\end{array}$ & $\begin{array}{l}10 \mathrm{M} \\
\text { Elite football players } \\
\text { Training experience: } \\
6 \text { training sessions/wk }\end{array}$ & $\begin{array}{l}\text { PBMC: WBC } \\
\text { CRP } \\
\text { CK }\end{array}$ & $\begin{array}{l}\text { After, at the end, } \\
\text { and daily for } 3 \\
\text { days after the exer }\end{array}$ & $\begin{array}{l}\uparrow C R P \text { at the end to } 1 \text { day and after } \\
\text { returns to baseline } \\
\uparrow C K \text { at } 2 \text { days and after returns to } \\
\text { baseline } \\
\leftrightarrow \text { WBC }\end{array}$ \\
\hline & & $\begin{array}{l}\text { Moderate: Resistance } \\
\text { training: squat, seated leg } \\
\text { extension, horizontal leg } \\
\text { curls, barbell side lunges, } \\
\text { and calf raises } \\
\text { (65-70\% } 1 \text { RM) } \\
4 \text { sets, } 8-10 \text { repetitions } \\
\text { per set with } 1 \text { min rest } \\
\text { Training during around } \\
40-45 \text { min following of } \\
10 \text { min warm-up }\end{array}$ & & & & $\begin{array}{l}\uparrow W B C \text { and CRP at } 1 \text { day and after } \\
\text { returns to baseline } \\
\text { Higher elevation in CRP, at the end } \\
\text { and } 24 \mathrm{~h} \text { after exer } \\
\uparrow C K \text { at } 1 \text { day and after returns } \\
\text { to baseline }\end{array}$ \\
\hline $\begin{array}{l}\text { Marklund et al. } \\
\text { (2013) }\end{array}$ & Cross-sectional & $\begin{array}{l}\text { Moderate: } 24 \mathrm{~h} \\
\text { ultra-endurance exer: } \\
\text { running, cycling, and } \\
\text { kayaking } \\
\left(46-63 \% \mathrm{VO}_{2 \mathrm{max}}\right) \text {; } \\
12 \text { sets of } 110 \text { min of exer } \\
\text { with } 10 \text { min rest for } \\
\text { food intake }\end{array}$ & $\begin{array}{l}9 \mathrm{M} \\
\text { Well-trained } \\
\text { ultra-endurance } \\
\text { athletes } \\
\text { Training experience: } \\
\text { competed in races } \\
\text { with long } \\
\text { distance (>48 h) }\end{array}$ & $\begin{array}{l}\text { PBMC: WBC } \\
\text { Cytokines: IL-6, } \\
\text { IL-8, IL-1 } 1 \beta \text {, and } \\
\text { TNF- } \alpha \\
\text { CRP } \\
\text { CK }\end{array}$ & $\begin{array}{l}\text { Before, at the end } \\
\text { and } 28 \mathrm{~h} \text { after the } \\
\text { exer }\end{array}$ & $\begin{array}{l}\uparrow W B C, I L-6 \text { and } C K \text { at the end and } \\
\downarrow 28 \mathrm{~h} \text { after exerc } \\
\uparrow I L-8 \text { and CRP at the end and } \\
\text { further } \uparrow \text { at } 28 \mathrm{~h} \\
\leftrightarrow \text { TNF- } \alpha \text { and IL- } 1 \beta\end{array}$ \\
\hline Nieman et al. (2012) & Cross-sectional & $\begin{array}{l}\text { Intense: } 1.75 \mathrm{~h} \text { cycling } \\
\text { followed by } 10 \mathrm{~km} \text { time trial } \\
\text { as fast as possible ( } 18.3 \pm \\
1.7 \mathrm{~min}) \text { total of } 2.1 \mathrm{~h} \text { cycling } \\
\text { (Borg scale }=13.3 \pm 1.1 \\
\text { and } 82.2 \pm 6.1 \% \mathrm{HR} \text { max })\end{array}$ & $\begin{array}{l}31 \mathrm{M} \\
\text { Trained cyclists } \\
\text { Training experience: } \\
\text { cycling } 75 \mathrm{~km}\end{array}$ & $\begin{array}{l}\text { PBMC: WBC and } \\
\text { lymphocytes } \\
\text { Cytokines: TNF-a, } \\
\text { IL-6, IL-8, IL-10, } \\
\text { and IL-1 } \beta\end{array}$ & $\begin{array}{l}\text { Before, at the end } \\
\text { and } 1 \mathrm{~h} \text { post-exer }\end{array}$ & $\begin{array}{l}\uparrow W B C \text { and lymphocyte at the end } \\
\text { and } \downarrow 1 \mathrm{~h} \text { after exer } \\
\uparrow T N F-\alpha, I L-6, I L-8, I L-10 \text {, and } \\
\text { IL- } 1 \beta \text { at the end of exer } \\
\downarrow T N F-\alpha, I L-6 \text {, and IL- } 81 \mathrm{~h} \text { after; } \\
\mathrm{IL}-10 \text { and IL- } 1 \beta \text { continued to } \\
\uparrow 1 \mathrm{~h} \text { after }\end{array}$ \\
\hline $\begin{array}{l}\text { Spiropoulos et al. } \\
\text { (2010) }\end{array}$ & Cross-sectional & $\begin{array}{l}\text { Intense: Ultra-endurance } \\
\text { foot race over a distance of } \\
246 \mathrm{~km}(9.08 \mathrm{MET} \text { by } \\
\text { middle age) } \\
\text { Finished the race in }<36 \mathrm{~h}\end{array}$ & $\begin{array}{l}10 \mathrm{M} \\
\text { Training experience: } \\
\text { done an equal } \\
\text { race before }\end{array}$ & $\begin{array}{l}\text { PBMC: WBC } \\
\text { Cytokines: IL-6; } \\
\text { CRP }\end{array}$ & $\begin{array}{l}\text { Before, at the end } \\
\text { and } 2 \text { days after } \\
\text { race }\end{array}$ & $\begin{array}{l}\uparrow W B C, I L-6 \text { and } C R P \text { at the end of } \\
\text { exer, IL- } 6 \text { and WBC return to } \\
\text { baseline } 2 \text { days after but CRP still } \uparrow\end{array}$ \\
\hline $\begin{array}{l}\text { Fatouros et al. } \\
(2006)^{\star}\end{array}$ & Cross-sectional & $\begin{array}{l}\text { Intense: } 4 \times 3 \text { wk resistance } \\
\text { training period divided in t1, } \\
\text { t2, t3, and t4 } \\
\text { t1 and t4: low-volume } \\
70 \% 1 \mathrm{RM}(\mathrm{t} 1 \text { and } \mathrm{t} 4) \text {, } \\
75-85 \% 1 \mathrm{RM} \text { (t2) and } \\
85-100 \% 1 \mathrm{RM} \text { (t3); } \\
2 \text { times per wk, } 2 \text { sets per } \\
\text { exer, } 10-12 \text { repeats per set } \\
\text { t2: high-volume training } \\
\text { ( } 4 \text { times per wk, } 4 \text { sets per } \\
\text { exer, 6-10 repeats per set) } \\
\text { t3: very-high-volume training } \\
\text { (6 times per wk, } 6 \text { sets per } \\
\text { exer, } 1-6 \text { repeats per set); }\end{array}$ & $\begin{array}{l}17 \mathrm{M} \\
\text { Amateur athletes }\end{array}$ & $\begin{array}{l}\text { CRP } \\
\text { CK }\end{array}$ & $\begin{array}{l}\text { Before and } 96 \mathrm{~h} \\
\text { after each session } \\
\text { of exer }\end{array}$ & $\begin{array}{l}\uparrow C R P \text { after } \mathrm{t} 2 \text { and } \mathrm{t} 3 \\
\uparrow \mathrm{CK} \text { after } \mathrm{t} 3 \text { and } \mathrm{t} 4\end{array}$ \\
\hline
\end{tabular}


TABLE 2 | Continued

\begin{tabular}{|c|c|c|c|c|c|c|}
\hline References & Study type & $\begin{array}{l}\text { Intensity and description } \\
\text { of exercise }\end{array}$ & Subjects & $\begin{array}{l}\text { Inflammatory } \\
\text { markers }\end{array}$ & Measure points & Findings \\
\hline $\begin{array}{l}\text { Degerstrøm and } \\
\text { Østerud (2006) }\end{array}$ & Cross-sectional & $\begin{array}{l}\text { Intense: } 2 \text { sets of } 30 \text { min run } \\
\text { with } 4 \mathrm{~h} \text { rest } \\
\left(80 \% \mathrm{VO}_{2 \max }\right)\end{array}$ & $\begin{array}{l}7 \mathrm{M} \\
5 \text { elite skiers and } 3 \\
\text { competing at the } \\
\text { district level } \\
\text { Training experience: } \\
1 \text { to } 2 \text { times/day }\end{array}$ & $\begin{array}{l}\text { PBMC: WBC and } \\
\text { lymphocytes } \\
\text { Cytokines: IL-6 } \\
\text { and IL-8 }\end{array}$ & $\begin{array}{l}\text { Before, at the end } \\
\text { each run and } 2 \mathrm{~h} \\
\text { after second run }\end{array}$ & $\begin{array}{l}\text { WBC: } \\
\uparrow \text { at the end 1st race; } \\
\downarrow \text { before 2nd race; } \\
\uparrow \text { more at the end 2nd race than } \\
\text { 1st; } \\
\downarrow 2 \mathrm{~h} \text { after 2nd race; } \\
\text { Lymphocytes and IL-8 } \uparrow \text { after 1st } \\
\text { and 2nd run, and IL-8 } \downarrow 2 \mathrm{~h} \text { after } \\
\text { 2nd race } \\
\leftrightarrow \mathbb{L}-6\end{array}$ \\
\hline $\begin{array}{l}\text { Connolly et al. } \\
\text { (2004) }\end{array}$ & Controlled Trial & $\begin{array}{l}\text { Intense: } 30 \text { min cycling } \\
\left(80 \% \mathrm{VO}_{2 \max }\right)\end{array}$ & $\begin{array}{l}15 \mathrm{M} \\
\text { Amateur athletes }\end{array}$ & $\begin{array}{l}\text { PBMC: WBC and } \\
\text { lymphocytes } \\
\text { Cytokines: IL-6 }\end{array}$ & $\begin{array}{l}\text { Before, at the end } \\
\text { and } 1 \mathrm{~h} \text { after exer }\end{array}$ & $\begin{array}{l}\uparrow W B C \text { and lymphocytes at the end } \\
\text { of exer and return to baseline } \\
\uparrow L-61 \mathrm{~h} \text { after exer }\end{array}$ \\
\hline Mucci et al. (1999) & Controlled Trial & $\begin{array}{l}\text { Intense: } \\
\left.\text { ( } 75 \text { and } 100 \% \text { of } \mathrm{VO}_{2 \max }\right) ; \\
\text { Moderate: } \\
\left(50 \% \text { of } \mathrm{VO}_{2 \max }\right) \text {; } \\
\text { Cycling at } 30 \text { Watts for } \\
\text { untrained and } 60 \text { watts for } \\
\text { trained athletes with } \\
\text { successive increases of } 30 \\
\text { watts every minute (at the } \\
\text { end of test the increase } \\
\text { was smaller) }\end{array}$ & $\begin{array}{l}22 \mathrm{M} \\
11 \text { highly trained } \\
\text { endurance athletes } \\
\text { (athletes group) } \\
\text { Training experience: } \\
5.2 \pm 06 \text { yr and } 16.3 \\
\pm 1 \mathrm{~h} / \mathrm{wk}\end{array}$ & $\begin{array}{l}\text { Cytokines: IL-1 } \beta \\
\text { and IL-8 }\end{array}$ & $\begin{array}{l}\text { Before the exer, at } \\
50 \% \mathrm{VO}_{2 \max }, 75 \% \\
\mathrm{VO}_{2 \max }, 100 \% \\
\mathrm{VO}_{2 \max } \text { and } \\
\text { recovery ( } 5 \text { min } \\
\text { after) }\end{array}$ & $\begin{array}{l}\uparrow I L-1 \beta \text { both intensities and returns to } \\
\text { baseline in recovery } \\
\uparrow I L-8 \text { both intensities and returns to } \\
\text { baseline in recovery }\end{array}$ \\
\hline & & $\begin{array}{l}\text { Upper limit of moderate: } 2 \mathrm{~h} \\
\text { cycling } \\
\left(60-65 \% \mathrm{VO}_{2 \max }\right)\end{array}$ & & & & $\begin{array}{l}\uparrow N K \text { cells at the end of exer and } \\
\text { return to baseline } 3 \mathrm{~h} \text { after } \\
\uparrow \text { Citolytic activity at the end of exer } \\
\uparrow I L-6 \text { at the end to } 3 \mathrm{~h} \text { after exer } \\
\uparrow T N F-\alpha \text { from } 3 \mathrm{~h} \text { to } 3 \text { days after } \\
\text { exer } \\
\leftrightarrow \mathrm{L}-10\end{array}$ \\
\hline & & $\begin{array}{l}\text { Moderate: } 3 \text { sets of } 10 \\
\text { repeats in resistance } \\
\text { training (bicep curl, knee } \\
\text { extension, hamstring, curl, } \\
\text { bench press, and leg press) } \\
\text { (60-70\% 1 RM) }\end{array}$ & & & & $\begin{array}{l}\uparrow N K \text { cells at the end of exer and } \\
\text { return to baseline } 3 \mathrm{~h} \text { after } \\
\uparrow \mathrm{CK} 3 \text { days after exer } \\
\leftrightarrow \mathrm{L}-10, \mathrm{IL}-6, \mathrm{LL}-10 \text { and } \mathrm{NK} \text { cells } \\
\text { cytolytic activity }\end{array}$ \\
\hline $\begin{array}{l}\text { Ostrowski et al. } \\
\text { (1999) }\end{array}$ & Cross-sectional & $\begin{array}{l}\text { Intense: Marathon (75.33\% } \\
\left.\mathrm{VO}_{2 \max }\right)\end{array}$ & $10 \mathrm{M}$ & $\begin{array}{l}\text { Cytokines: TNF- } \alpha \text {, } \\
\text { IL-1 } 1 \beta, \text { IL- } 6 \text { and } \\
\text { IL-10 }\end{array}$ & $\begin{array}{l}1 \mathrm{wk} \text { before, at the } \\
\text { end and every } \\
30 \text { min in the } 4 \mathrm{~h} \\
\text { after exer }\end{array}$ & $\begin{array}{l}\uparrow T N F-\alpha, I L-1 \beta, I L-6 \text { and IL-10 at the } \\
\text { end } \\
\downarrow I L-10 \text { and IL- } 6 \text { (immediately) } \\
\downarrow T N F-\alpha \text { and IL-1 } 1 \beta \text { (slowly) }\end{array}$ \\
\hline
\end{tabular}

$\uparrow$, significant increase; $\downarrow$, significant decrease; $\leftrightarrow$, no change; 1 RM, one repeat maximum; 1st, first; 2nd, second; CK, creatinine kinase; CRP, C-reactive protein; Exer, exercise; F, Female; $h$, hour; HIGH, high intensity; HM, half marathon; HRmax, maximum heart rate; IL, interleukin; LV-HIIE, high intensity interval exercise; M, male; PBMC, peripheral blood mononuclear cel; TNF- $\alpha$, Tumor necrosis factor alpha; $V$, velocity; VO $\mathrm{O}_{\text {max }}$, maximum rate of oxygen consumption; WBC, white blood cells; wk, week; yr, years; * $<75 \%$ in STROBE quality scale; " < $75 \%$ in CONSORT quality scale. 
TABLE 3 | Immediate effects of moderate and intense exercise (0-15 min) on inflammatory markers.

\begin{tabular}{|c|c|c|c|c|}
\hline Inflammatory marker & Moderate exercise & References & Intense exercise & References \\
\hline \multirow[t]{2}{*}{ WBC } & $\leftrightarrow$ & $\begin{array}{l}\text { Draganidis et al., 2013; } \\
\text { Marklund et al., } 2013\end{array}$ & $\begin{array}{l}\uparrow] 1.44 ; 3.5[x \\
d=9.28 \pm 6.15^{\S}\end{array}$ & $\begin{array}{l}\text { Bonsignore et al., 2001; } \\
\text { Connolly et al., 2004; } \\
\text { Degerstrøm and Østerud, 2006; } \\
\text { Spiropoulos et al., 2010; } \\
\text { Nieman et al., 2012; } \\
\text { Gonzalo-Calvo et al., 2015; } \\
\text { Stelzer et al., } 2015\end{array}$ \\
\hline & & & $\leftrightarrow$ & $\begin{array}{l}\text { Bernecker et al., 2011; } \\
\text { Abbasi et al., 2013; } \\
\text { Draganidis et al., } 2013\end{array}$ \\
\hline \multirow[t]{3}{*}{ Lymphocytes } & $\begin{array}{l}\uparrow 1.41 \times \\
d=1.98\end{array}$ & Wadley et al., 2015 & $\begin{array}{l}\uparrow] 1.30 ; 2.69[x \\
d=6.52 \pm 4.26\end{array}$ & $\begin{array}{l}\text { Connolly et al., 2004; } \\
\text { Degerstrøm and Østerud, 2006; } \\
\text { Nieman et al., 2012; } \\
\text { Gonzalo-Calvo et al., 2015; } \\
\text { Stelzer et al., 2015; } \\
\text { Wadley et al., } 2015\end{array}$ \\
\hline & & & $\begin{array}{l}\downarrow 4.21 x \\
d=3.89\end{array}$ & Bonsignore et al., 2001 \\
\hline & & & $\leftrightarrow$ & Abbasi et al., 2013 \\
\hline NK cells & $\begin{array}{l}\uparrow 5.5 x \\
d=8.20\end{array}$ & Brenner et al., 1999 & $\begin{array}{l}\uparrow 8.83 x \\
d=10.12\end{array}$ & Brenner et al., 1999 \\
\hline NK cells cytolytic activity & $\begin{array}{l}\uparrow 2.92 \times \\
d=10.48\end{array}$ & Brenner et al., 1999 & $\begin{array}{l}\uparrow 4.63 x \\
d=13.86\end{array}$ & Brenner et al., 1999 \\
\hline \multirow[t]{2}{*}{ IL-6 } & $\begin{array}{l}\uparrow] 1.33 ; 4.20[x \\
d=3.91 \pm 3.87\end{array}$ & $\begin{array}{l}\text { Brenner et al., 1999; } \\
\text { Wadley et al., } 2015\end{array}$ & $\begin{array}{l}\uparrow] 1.59 ; 26.79[x \\
d=4.04 \pm 2.34 \S\end{array}$ & $\begin{array}{l}\text { Ostrowski et al., 1999; } \\
\text { Connolly et al., 2004; } \\
\text { Spiropoulos et al., 2010*; } \\
\text { Nieman et al., } \\
\text { 2012; Gonzalo-Calvo et al., 2015; } \\
\text { Stelzer et al., 2015; } \\
\text { Ulven et al., 2015; } \\
\text { Wadley et al., 2015 }\end{array}$ \\
\hline & $\leftrightarrow$ & $\begin{array}{l}\text { Marklund et al., 2013; } \\
\text { Azizbeigi et al., } 2015\end{array}$ & $\leftrightarrow$ & $\begin{array}{l}\text { Brenner et al., 1999; } \\
\text { Degerstrøm and Østerud, 2006; } \\
\text { Bernecker et al., 2011; } \\
\text { Azizbeigi et al., } 2015\end{array}$ \\
\hline \multirow[t]{2}{*}{ IL-10 } & $\leftrightarrow$ & $\begin{array}{l}\text { Brenner et al., 1999; } \\
\text { Wadley et al., } 2015\end{array}$ & $\begin{array}{l}\uparrow] 1.57 ; 32.99[x \\
d=4.55 \pm 3.37\end{array}$ & $\begin{array}{l}\text { Ostrowski et al., 1999; } \\
\text { Nieman et al., 2012; } \\
\text { Gonzalo-Calvo et al., 2015; } \\
\text { Ulven et al., } 2015\end{array}$ \\
\hline & & & $\leftrightarrow$ & $\begin{array}{l}\text { Brenner et al., 1999; } \\
\text { Wadley et al., } 2015\end{array}$ \\
\hline \multirow[t]{2}{*}{ IL-8 } & $\begin{array}{l}\uparrow 1.43 x \\
d=1.04\end{array}$ & Mucci et al., 1999 & $\begin{array}{l}\uparrow] 1.37 ; 2.77[x \\
d=3.63 \pm 2.41\end{array}$ & $\begin{array}{l}\text { Mucci et al., 1999; } \\
\text { Degerstrøm and Østerud, 2006; } \\
\text { Nieman et al., 2012; } \\
\text { Gonzalo-Calvo et al., } 2015\end{array}$ \\
\hline & $\leftrightarrow$ & Marklund et al., 2013 & & \\
\hline \multirow[t]{2}{*}{$\mathrm{IL}-1 \beta$} & $\begin{array}{l}\uparrow 1.13 x \\
d=0.64\end{array}$ & Mucci et al., 1999 & $\begin{array}{l}\uparrow] 1.13 ; 1.50[x \\
d=0.58 \pm 0.29\end{array}$ & $\begin{array}{l}\text { Mucci et al., 1999; } \\
\text { Ostrowski et al., 1999; } \\
\text { Nieman et al., } 2012\end{array}$ \\
\hline & $\leftrightarrow$ & Marklund et al., 2013 & & \\
\hline \multirow[t]{2}{*}{ TNF- $\alpha$} & $\leftrightarrow$ & $\begin{array}{l}\text { Brenner et al., 1999; } \\
\text { Marklund et al., 2013; } \\
\text { Azizbeigi et al., } 2015\end{array}$ & $\leftrightarrow$ & $\begin{array}{l}\text { Brenner et al., 1999; } \\
\text { Bernecker et al., 2011; } \\
\text { Azizbeigi et al., } 2015\end{array}$ \\
\hline & & & $\begin{array}{l}\uparrow] 1,30 ; 2.07[x \\
d=2.96 \pm 2.20\end{array}$ & $\begin{array}{l}\text { Ostrowski et al., 1999; } \\
\text { Nieman et al., 2012; } \\
\text { Ulven et al., } 2015\end{array}$ \\
\hline
\end{tabular}


TABLE 3 | Continued

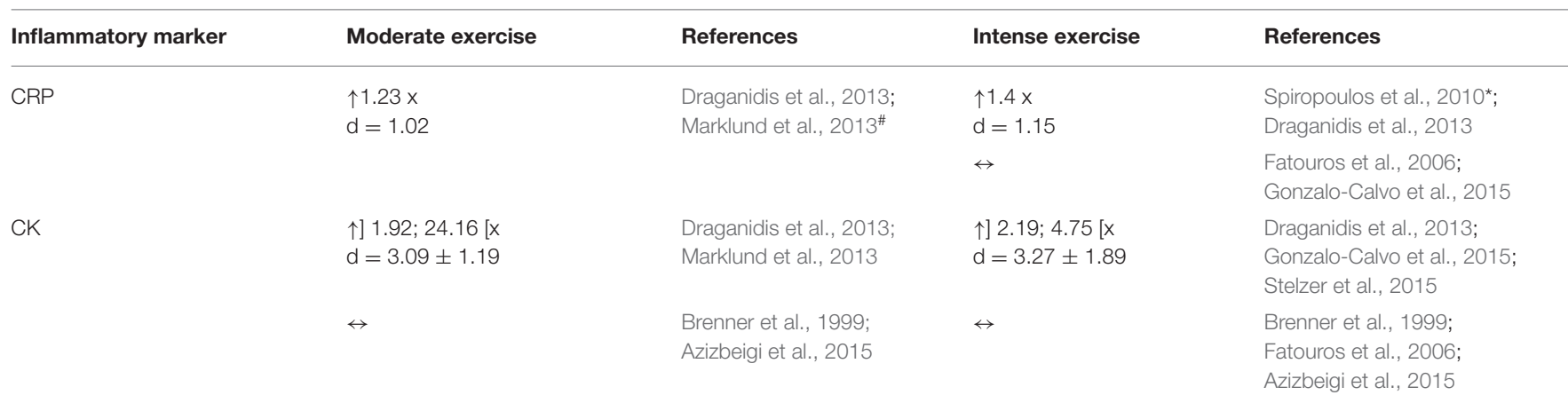

$\uparrow$, significant increase; $\downarrow$, significant decrease; $\leftrightarrow$, no change; CRP, C-reactive protein; $d$, effect size, expressed as mean \pm sd; IL, Interleukin; TNF- $\alpha$, tumor necrosis factor alpha; WBC, white blood cells; *Spiropoulos et al. was not included to calculate the increase interval of IL-6 and CRP due very discrepant values after exercise when compared to the other studies

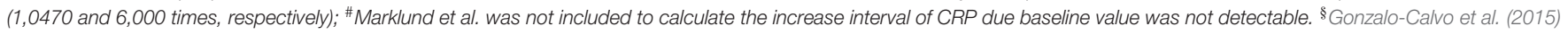
and Ostrowski et al. (1999). were not included to calculate $d$ due very discrepant value of 53.03 and 53.18, respectively.

cytokine levels after moderate and intense exercise but only show an increase in IL-6 after moderate exercise (recovery).

Some studies that evaluated only intense exercise showed an increase in IL-6, IL-8, and IL-10; however, values peaked at different times: immediately $(15 \mathrm{~min})$ for IL-6 and IL-10 in Ostrowski et al. (1999), 1 or $2 \mathrm{~h}$ after exercise for IL-6, IL-8, and IL-10 in Nieman et al. (2012), and for IL-8 in Degerstrøm and Østerud (2006). Marklund et al. (2013) that evaluated moderate ultra-endurance exercise showed an increase in IL-8 and IL-6, $30 \mathrm{~min}$ after exercise, however only for IL-8 did this increase remained at $28 \mathrm{~h}$. Spiropoulos et al. (2010) evaluated an intense ultra-endurance exercise and observed an increase of IL6 maintained at 2 days. The same was observed for the studies by Gonzalo-Calvo et al. (2015), Ostrowski et al. (1999), and Bernecker et al. (2011). These discrepancies might be explained by the duration of the exercises in those studies: the Spiropoulos study refers to ultra-endurance exercise, and the remaining three studies to marathon races (Ostrowski et al., 1999; Bernecker et al., 2011; Gonzalo-Calvo et al., 2015).

Globally the increase in IL-6 and IL-8 levels was higher in intense when compared to moderate exercises. In contrast, IL10 only showed increases after intense exercise, with no changes after moderate exercise (Figures S1, S2). Nevertheless, the impact of the duration of the exercise bout should be considered when comparing the studies' results.

IL-1 $\beta$ was evaluated in 4 studies with discrepant results (Mucci et al., 1999; Ostrowski et al., 1999; Nieman et al., 2012; Marklund et al., 2013). Mucci et al. (1999) reported approximately the same increase and return to baseline in both exercise intensities for IL$1 \beta$. The same pattern was reported in intense exercise by Nieman et al. (2012) and Ostrowski et al. (1999). In contrast, Marklund et al. (2013) reported no changes in this cytokine.

TNF- $\alpha$ was evaluated in 8 studies corresponding to three moderate exercise types without alteration (Brenner et al., 1999; Marklund et al., 2013; Azizbeigi et al., 2015) and six intense exercise types: three with no alteration on the cytokine levels (Brenner et al., 1999; Bernecker et al., 2011; Azizbeigi et al., 2015) and three with increases immediately after exercise (Ostrowski et al., 1999; Nieman et al., 2012; Ulven et al., 2015). All the studies reporting alterations had exercise times of more than $1 \mathrm{~h}$. Only the study by Ostrowski et al. (1999) had repetitive measurements showing a slow decrease in TNF- $\alpha$ values without returning to base levels after $4 \mathrm{~h}$.

\section{Effects of Exercise on Peripheral Blood Leukocytes}

The number of WBC increased after intense exercise in 7 studies (Bonsignore et al., 2001; Connolly et al., 2004; Degerstrøm and Østerud, 2006; Spiropoulos et al., 2010; Nieman et al., 2012; Gonzalo-Calvo et al., 2015; Stelzer et al., 2015). Abbasi et al. (2013) evaluated the effect of high-intensity exercise and observed an increase in WBC numbers $30 \mathrm{~min}$ after exercise. This elevation was maintained even after a $3 \mathrm{~h}$ recovery period and reflected a pronounced granulocytosis. In contrast, total lymphocyte count had no alterations in the same period but increased $30 \mathrm{~min}$ after exercise. All leukocyte counts returned to normal $24 \mathrm{~h}$ post-exercise (Abbasi et al., 2013). Similar to this study, Bonsignore et al. (2001) showed an increase of WBC and a decrease of lymphocytes. This increase in WBC occurred due to neutrophils and macrophages.

Nieman et al. (2012) that analyzed the influence of prolonged cycling in high intensity in acute inflammatory response showed an increase in WBC, but, in contrast to previous studies, this occurred due to increased lymphocytes, monocytes, and granulocytes. Lymphocytes and WBC returned to baseline $1 \mathrm{~h}$ after exercise. The same pattern occurred in other 3 studies: Degerstrøm and Østerud (2006) with increases at $2 \mathrm{~h}$ after exercise, Connolly et al. (2004) with increases at $1 \mathrm{~h}$ after exercise and Stelzer et al. (2015) with increases immediately after exercise. These increases in WBC were reportedly due to lymphocytes and granulocytes in the Degerstrøm and Østerud (2006) study; due to neutrophils, monocytes and lymphocytes in the Stelzer et al. (2015) study; and due to lymphocytes and monocytes in the Connolly et al. (2004) study. Other 2 studies of intense exercise also evaluate WBC showing increases after exercise but do not have data on relevant subpopulations (Spiropoulos et al., 2010; Bernecker et al., 2011). 
Draganidis et al. (2013) analyzed WBC after resistance training (intense and moderate exercise) showing an increase after moderate exercise, which returns to baseline 1 day after and did not report alterations in WBC after intense exercise. Wadley et al. (2015) evaluated lymphocytes but not total WBC after cycling exercise (moderate and intense) and reported an increase in both intensities that returns to baseline $30 \mathrm{~min}$ after exercise. Marklund et al. (2013) evaluated WBC 30 min after moderate exercise showing an increase with a return to baseline values $28 \mathrm{~h}$ after exercise.

When comparing both exercise intensities, the increase in total leukocytes only occurs after intense exercise. However, in the lymphocytes subpopulation, both intensities showed similar increases, with only one study presenting a decrease in lymphocytes after intense exercise (Bonsignore et al., 2001).

One study specifically considered NK cell numbers and NK cytolytic activity: Brenner et al. (1999) showed an increase in both parameters after exercise. This increase was greater in intense exercise when compared to moderate exercise (Brenner et al., 1999).

\section{Effect of Exercise on CK}

$\mathrm{CK}$ was evaluated in 7 studies corresponding to 10 different exercise types. In short, CK increased in 4 intense type exercises (Fatouros et al., 2006; Draganidis et al., 2013; Gonzalo-Calvo et al., 2015; Stelzer et al., 2015) and 2 moderate exercises (Draganidis et al., 2013; Marklund et al., 2013), with no alteration in the remainder studies evaluating moderate and intense exercise in the same volunteer group. In the study of Draganidis et al. (2013) the increase in CK peaked at $24 \mathrm{~h}$ after exercise in the moderate-intensity group, while it peaked at $48 \mathrm{~h}$ in the highintensity group. However, in the study by de Gonzalo-Calvo et al. (2015), CK peaked $24 \mathrm{~h}$ after intense exercise and in the study by Marklund et al. (2013) $28 \mathrm{~h}$ after moderate exercise. Fatouros et al. (2006) evaluated CRP 4 days after exercise showing an increase. Comparing intense and moderate exercise, the increase in CK was greater in moderate exercise, but only 2 studies evaluate this intensity when compared to 4 studies evaluating the intense exercise.

\section{Effect of Exercise on CRP}

Increases in CRP were observed in one study of moderate exercise (Draganidis et al., 2013) and two studies of intense exercise (Fatouros et al., 2006; Draganidis et al., 2013). In the Draganidis et al. (2013) study the elevation was maintained for 1-2 days before returning to baseline. The increase in CRP on Fatouros et al. (2006) only shows results immediately after exercise. Marklund et al. (2013) evaluating a moderate exercise type, showed an elevation of CRP $30 \mathrm{~min}$ after exercise with greater increases $28 \mathrm{~h}$ after, but the ratio of the increase cannot be calculated due to the lack of the baseline value. GonzaloCalvo et al. (2015) reported elevations of CRP at $24 \mathrm{~h}$ after exercise, without alterations before that. Globally, all the studies had increases of this inflammatory marker, with greater values at $24 \mathrm{~h}$. Comparing both exercise intensities, the increase was greater after intense exercise, but only one study referred to moderate-intensity exercise.

\section{DISCUSSION}

This systematic review evaluated the changes of inflammatory markers after moderate and intense exercise bouts. The findings of the current review suggest that there is an acute inflammation profile after exercise, with the increase of most inflammatory markers, especially in high-intensity exercise. In samples taken immediately after long and intense exercises we could not rule out the effects of dehydration on the plasma volume, and hence the quantification of the inflammatory markers measured.

\section{Exercise-Induced Effects on Cytokines}

Results suggested that there are substantial discrepancies in the extent of pro-inflammatory changes in the immune system. By examining the impact of different intensity exercise on antiinflammatory cytokines secretion, some of the studies showed an increase while others report no change (Brenner et al., 1999; Bernecker et al., 2011; Marklund et al., 2013; Azizbeigi et al., 2015). This might be a consequence of cytokines appearing only transiently in the blood and thereby evading detection. Moreover, cytokines are secreted by many cells and tissues, with muscle considered to be a major contributor during exercise, as such, circulating levels might not reflect levels in source tissues.

IL-6 concentration increases more than other cytokines during exercise which might indicate muscle damage (Allen et al., 2015; Lightfoot and Cooper, 2016). IL-6 plasma concentrations are reportedly affected by factors other than intensity, such as type and time of exercise (Gleeson et al., 2011; Baumert et al., 2016). Persistent elevation of this cytokine can be associated with muscle atrophy, that results in a reduction on strength and muscle function and increased muscle pain (Hennigar et al., 2017). Following acute exercise, elevated levels of IL-6 promote an increase in IL-10 and IL-1RA, two antiinflammatory cytokines.

Most studies showed an increase in IL-10 after intense exercise which is consistent with previous reports (Moldoveanu et al., 2001; Allen et al., 2015). Shaw et al. (2017) demonstrated that IL10 production, after strenuous acute exercise, is equivocal, with increases, decreases and no changes in exercises with different protocols and analytical techniques. Regardless, levels of IL10 tend to peak during recovery time from exercise, with the magnitude of the increase being related to the active muscle mass and exercise intensity. Overall, the duration of the exercise was the most important factor determining the magnitude of the exercise-induced increase of plasma IL-10, as recently reviewed by Santos et al. (2019). This increase in IL-10 could be related to the prevention of potential deleterious chronic low-grade inflammation and tissue damage.

TNF- $\alpha$ was only stimulated by intense endurance exercise (more than 1 h) (Ostrowski et al., 1999; Nieman et al., 2012; Ulven et al., 2015). In contrast to our main findings, Starkie et al. (2003) showed that the production of TNF- $\alpha$ decreased after a single endurance exercise bout and Moldoveanu et al. (2001) reported an increase in TNF- $\alpha$ after three $2-3 \mathrm{~h}$ moderate bouts (cycling and run + cycling). However, no change was found after a single $45 \mathrm{~min}$ moderate bout or one $5 \mathrm{~min}$ intense bout (Moldoveanu 
et al., 2001). These results suggest that other factors, namely exercise duration, are also important in regulating TNF- $\alpha$ release.

Previous reports suggested that IL-1 release depends on the type, intensity and duration of exercise (Moldoveanu et al., 2001). The current review shows that IL- $1 \beta$ increases in all studies of intense exercise but in none after moderate exercise. Similar to TNF- $\alpha$, the local increase of IL- 1 is higher than the systemic increase, after eccentric exercise (Baumert et al., 2016). IL-1 $\beta$ is a potent pro-inflammatory cytokine, influencing adhesion molecules and chemokines, and by this, possibly relating to leukocyte migration and function.

In this systematic review, it was observed that IL-8 increased after both exercise intensities, which is consistent with previous reviews that report increased IL-8 systemic levels associated with damaging exercise regimes (Moldoveanu et al., 2001; Lightfoot and Cooper, 2016). Suzuki et al. (2003) showed that IL-8 increases after prolonged exercise, with some alteration after acute intense exercise. The same occurs in this systematic review, with IL-8 systemic levels increasing after prolonged and acute intense exercises.

In general, cytokines increase more with intense than with moderate exercise, but these increases are not consistent, being influenced by the duration and type of exercise.

\section{Exercise-Induced Effects on WBC}

Peake et al. (2017) showed that a single bout can cause changes in blood number of leukocytes, which persist during exercise recovery and in contrast, decrease faster (in $30 \mathrm{~min}$ ) after especially prolonged and/or intense exercise. The included studies showed a WBC increase immediately after intense exercise; however, in the recovery period, the decrease was inconsistent between studies. WBC number was the only inflammatory marker studied that showed a clear increase after intense exercise in all the included studies, without alteration in moderate exercise studies. The results also suggest a chronology in the mobilization of the different leukocyte populations to the blood, with lymphocytosis occurring at the end of the intense exercise bouts and decreasing shortly after $(30 \mathrm{~min})$ (Wadley et al., 2015).

As a part of the innate immune system, NK cells can recognize and eliminate neoplastic and virus-infected cells without prior contact (Bigley and Simpson, 2015). The decrease in NK activity is accompanied by an increased incidence of infectious diseases (Fu et al., 2014). NK cell number and NK cell activity increased after moderate and intense exercise, but this was verified in only one study included in this systematic review. The study by Brenner et al. (1999) showed that acute physical exercise increased the activity and induced mobilization of NK cells to the peripheral blood independently of exercise intensity. Theoretically, a high frequency of NK cells can possibly protect the body against infections or tumor progression, but it must be bared in mind that these increases were transitory, and possible migration of NK cells to peripheral tissues was not assessed. Previous reviews reported NK and NK activity increases in response to stressors (Timmons and Cieslak, 2008; Viana et al., 2014; Bigley and Simpson, 2015; Peake et al., 2017).
Studies that explore the true effect of changes in cell distribution in response to exercise and health status are lacking, and the relevance of these findings cannot be fully appraised as we failed to consider the number of cells infiltrating in the muscle tissue in response to exercise. In fact, Marklund et al. (2013) showed that a moderate-intensity endurance activity ( $\sim 60 \%$ V02 peak) sustained during a very prolonged period induced an extensive local muscle infiltration.

\section{Exercise-Induced Effects on CK}

CK results were very discrepant, as half of the studies showed no alterations immediately after intense or moderate exercise, while the other half showed an increase of CK levels. MoghadamKia et al. (2016) referred that CK levels have a significant variation with sex and race. The degree of this change depended on the duration and type of exercise; with strenuous exercise being responsible for greater elevations. Damaged muscle fiber structures were pointed out as being the cause for the rise, but one study of repeated eccentric exercise caused almost no increase on CK levels (Baumert et al., 2016). CK was the only marker whose increase was higher in moderate when compared to intense exercise, but few studies were available in the moderate exercise arm. In general, the muscle damage, as evidenced by CK activity was not accompanied by parallel increases in inflammatory markers, namely cytokines and CRP.

\section{Exercise-Induced Effects on CRP}

Of the proteins stimulated during the acute phase response, CRP has received the most attention as a marker of inflammation in both rheumatic and non-rheumatic diseases (Petersen and Pedersen, 2005; Schrödl et al., 2016). It is proposed to have a scavenger function to eliminate bacterial products or damaged cells and to attenuate the consequences of infection or tissue injury. Petersen and Pedersen (2005) reported that this inflammatory marker has a role in the suppression of the synthesis of pro-inflammatory cytokines by tissue macrophages and in the induction of anti-inflammatory cytokines. Because the levels of CRP increase dramatically during inflammation processes and remain elevated for a long period of time CRP can be a suitable marker. In this systematic review, two studies (Fatouros et al., 2006; Draganidis et al., 2013) had an increase in CRP at the end of exercise in contrast of Petersen and Pedersen (2005) where CRP increased 1 day after. This discrepancy may be due to the fact that in those studies the athletes did short sets of exercise and in Petersen and Pedersen (2005) they practice longer duration exercises. Fedewa et al. (2016) indicated that CRP had a significant and small decrease following training, but doesn't report values immediately after acute exercise. This information is in opposition to Petersen and Pedersen (2005), showing that regular exercise induces a reduction in CRP.

\section{Implications for Practice}

Exercise has been established as a part of multimodal therapeutic approaches in several pathologies contributing to cardiorespiratory fitness, muscle strength, flexibility, and neuromotor performance. However, the strong variability in study designs, type, duration, and intensity of exercise remain 
obstacles in the assessment of the measurable effects of exercise on inflammatory markers.

A recent systematic review on the impact of physical activity on serum levels of inflammatory markers in rheumatoid arthritis patients failed to conclude that there is a significant impact on systemic levels of inflammatory markers (Burghardt, 2019). Nevertheless, attention is needed when recommending and prescribing physical activity to these specific patients. One should be aware of the possible influence of medication, and the potential increase of pain and disease activity by performing physical activity, even without any changes of inflammatory markers.

Previous studies suggested that the effect of aerobic exercise on the cytokines' increase is different from the effect of strength/resistance exercise, with the latter being less evident (Santos et al., 2019). This is in line with our evidence. Moreover, strength/resistance exercises are influenced by different variables such as intensity, workload, number of repetitions, the interval between sets, and size of muscle mass involved in muscle contraction. Whereas, the anti-inflammatory nature of IL-6 contributes to the acute phase response and the adaptation of skeletal muscle to exercise, chronically elevated levels of IL6 contribute to persistent inflammation and muscle wasting (Lightfoot and Cooper, 2016). The release of anti-inflammatory mediators, such as IL-10, as a compensatory mechanism, might also impair immune responses. The pronounced antiinflammatory response induced by prolonged and exhaustive exercise could lead to transient suppression of several immune components and increase the risk of infection (Shaw et al., 2017).

\section{Limitations}

The results of this review were based on individual sports, such as cycling, resistance training, and running, which limits its application to other types of sports. Some limitations were found in the compilation and comparison of results because the time, type of exercise, and a number of bouts were different among studies. In addition, we did not perform a comparative analysis (meta-analysis), because such analysis could not be easily accomplished due to the lack of consistency in parameters and the lack of uniformity. Because of the non-response of some study's authors, some articles with important findings might not be included. CRP was the most restrictive inflammatory marker, with no possible comparison of concentrations since measurement methods varied widely. Our findings showed that most studies follow the same pattern of changes; however, the amplitude of those changes at the systemic level does not always correlate with exercise-induced changes in local inflammation. Another limitation is that most of the studies performed the experiment at a single level of intensity with a relatively small number of participants, which might have contributed to increasing the individual variability. All the studies included in this systematic review refer to healthy non-sedentary individuals. As such, it is not possible to ascertain if the same results would be valid for sedentary individuals that initiate exercise practice and what would the implications be in populations with chronic inflammatory pathologies. Our conclusions might also have been limited by restricting our search to the PubMed database, as other relevant studies might have not been considered.

\section{CONCLUSION}

Based on the current review findings, exercise has considerable effects on inflammation markers. Pro-inflammatory cytokine TNF- $\alpha$ and anti-inflammatory IL-10 only increase after intense exercise, and pro-inflammatory cytokines IL-6 and IL- $1 \beta$ increase more with intense than with moderate exercise. The main differences regarding the effect of intensity of exercise on the inflammation markers studies were found in total WBC, IL6 , and IL-10, with higher increases in intense than in moderate exercise bouts. The highest alterations occur after intense exercise in IL-6 with increases up to 26.79 times and in IL-10 with increases up to 32.99 times, corresponding a $\mathrm{VO}_{2 \max }$ of $75.33 \%$ In moderate exercise studies, higher alterations occur in $\mathrm{CK}$ with an increase of 24.16 times at a $\mathrm{VO}_{2 \max }$ of $46-63 \%$. However, our results were not consistent, with discrepancies probably due to the emphasis on muscle contraction (eccentric vs. concentric) and intensity of the effort related to the type of the exercise. Nevertheless, and although regular exercise presents a global positive anti-inflammatory effect, high-intensity exercise, especially when performed with reduced recovery periods, induces a persistent dysregulation of the immune system with increased susceptibility to illness. Further research is required to examine the impact of exercise intensity on inflammation. It is important that future studies carefully assess not only intensity but also associate it with exercise type and duration, as those aspects were found to deeply influence inflammation within the intense exercise group.

\section{AUTHOR CONTRIBUTIONS}

ÉC and OL carried out literature searches and wrote the manuscript's first draft. $\mathrm{DM}$ and $\mathrm{HN}$ checked the sports aspects and the potential training implications. OL checked the immunological aspects. EC, DM, HN, and OL checked the final version of the manuscript. All authors made contributions to the review.

\section{FUNDING}

This work is supported by national funding through the Portuguese Foundation for Science and Technology, I.P., under the project UID/DTP/04045/2019 and the European Fund for Regional Development (FEDER) allocated by European Union through the COMPETE 2020 Programme (POCI-01-0145FEDER-006969) - competitiveness and internationalization.

\section{SUPPLEMENTARY MATERIAL}

The Supplementary Material for this article can be found online at: https://www.frontiersin.org/articles/10.3389/fphys. 2019.01550/full\#supplementary-material 


\section{REFERENCES}

Abbasi, A., Hauth, M., Walter, M., Hudemann, J., Wank, V., Niess, A. M., et al. (2013). Exhaustive exercise modifies different gene expression profiles and pathways in LPS-stimulated and un-stimulated whole blood cultures. Brain. Behav. Immun. 39, 130-141. doi: 10.1016/j.bbi.2013.10.023

Allen, J., Sun, Y., and Woods, J. A. (2015). Exercise and the regulation of inflammatory responses. Prog. Mol. Biol. Transl. Sci. 135, 337-354. doi: $10.1016 /$ bs.pmbts.2015.07.003

Azizbeigi, K., Azarbayjani, M. A., Atashak, S., and Stannard, S. R. (2015). Effect of moderate and high resistance training intensity on indices of inflammatory and oxidative stress. Res. Sport. Med. 23, 73-87. doi: 10.1080/15438627.2014.975807

Baumert, P., Lake, M. J., Stewart, C. E., Drust, B., and Erskine, R. M. (2016). Genetic variation and exercise-induced muscle damage: implications for athletic performance, injury and ageing. Eur. J. Appl. Physiol. 116, 1595-1625. doi: 10.1007/s00421-016-3411-1

Bernecker, C., Scherr, J., Schinner, S., Braun, S., Scherbaum, W. A., and Halle, M. (2011). Evidence for an exercise induced increase of TNF- $\alpha$ and IL-6 in marathon runners. Scand. J. Med. Sci. Sport. 23, 207-214. doi: 10.1111/j.1600-0838.2011.01372.x

Bigley, A. B., and Simpson, R. J. (2015). NK cells and exercise: implications for cancer immunotherapy and survivorship. Discov. Med. 19, 433-445.

Bonsignore, M. R., Morici, G., Riccobono, L., Insalaco, G., Bonanno, A., Profita, M., et al. (2001). Airway inflammation in nonasthmatic amateur runners. Am. J. Physiol. Lung Cell. Mol. Physiol. 281, L668-L676. doi: 10.1152/ajplung.2001.281.3.L668

Bonsignore, M. R., Morici, G., Riccobono, L., Insalaco, G., Bonanno, A., Profita, M., et al. (2013). Airway inflammation in nonasthmatic amateur runners. Mol. Immunol. 281, 129-134.

Brenner, I. K. M., Natale, V. M., Vasiliou, P., Moldoveanu, A. I., Shek, P. N., and Shephard, R. J. (1999). Impact of three different types of exercise on components of the inflammatory response. Eur. J. Appl. Physiol. Occup. Physiol. 80, 452-460. doi: $10.1007 /$ s004210050617

Burghardt, R. (2019). Markers in rheumatoid arthritis: a systematic literature review. Rheumatol. Int. 39, 793-804. doi: 10.1007/s00296-019-04284-x

Chen, L., Liu, H., Yuan, M., Lu, W., Wang, J., and Wang, T. (2018). The roles of interleukins in perfusion recovery after peripheral arterial disease. Biosci. Rep. 38:BSR20171455. doi: 10.1042/BSR20171455

Cohen, J. (1988). Statistical Power Analysis for the Behavioural Sciences. Hillsdale, MI: Erlbaum.

Connolly, P. H., Caiozzo, V. J., Zaldivar, F., Nemet, D., Larson, J., Hung, S.- P., et al. (2004). Effects of exercise on gene expression in human peripheral blood mononuclear cells. J. Appl. Physiol. 97, 1461-1469. doi: 10.1152 /japplphysiol.00316.2004

Degerstrøm, J., and Østerud, B. (2006). Increased inflammatory response of blood cells to repeated bout of endurance exercise. Med. Sci. Sports Exerc. 38, 1297-1303. doi: 10.1249/01.mss.0000227315.93351.8d

Draganidis, D., Chatzinikolaou, A., Jamurtas, A. Z., Carlos Barbero, J., Tsoukas, D., Theodorou, A. S., et al. (2013). The time-frame of acute resistance exercise effects on football skill performance: the impact of exercise intensity. J. Sports Sci. 31, 714-722. doi: 10.1080/02640414.2012.746725

Fatouros, I. G., Destouni, A., Margonis, K., Jamurtas, A. Z., Vrettou, C., Houretas, D., et al. (2006). Cell-free plasma DNA as a novel marker of aseptic inflammation severity related to exercise overtraining. Clin. Chem. 52, 1820-1824. doi: 10.1373/clinchem.2006.070417

Fedewa, M. V., Hathaway, E. D., and Ward-Ritacco, C. L. (2016). Effect of exercise training on $\mathrm{C}$ reactive protein: a systematic review and meta-analysis of randomised and non-randomised controlled trials. Br. J. Sports Med. 51, 670-676. doi: 10.1136/bjsports-2016-095999

Fu, B., Tian, Z., and Wei, H. (2014). Subsets of human natural killer cells and their regulatory effects. Immunology 141, 483-489. doi: 10.1111/imm.12224

Gleeson, M., Bishop, N. C., Stensel, D. J., Lindley, M. R., Mastana, S. S., and Nimmo, M. A. (2011). The anti-inflammatory effects of exercise: mechanisms and implications for the prevention and treatment of disease. Nat. Rev. Immunol. 11, 607-610. doi: 10.1038/nri3041

Gonzalo-Calvo, D., de, Dávalos, A., Montero, A., García-González, Á., Tyshkovska, I., González-Medina, A., et al. (2015). Circulating inflammatory miRNA signature in response to different doses of aerobic exercise. J. Appl. Physiol. 119, 124-134. doi: 10.1152/japplphysiol.00077.2015

Hennigar, S. R., McClung, J. P., and Pasiakos, S. M. (2017). Nutritional interventions and the IL-6 response to exercise. FASEB J. 31, 3719-3728. doi: $10.1096 /$ fj.201700080R

Lightfoot, A. P., and Cooper, R. G. (2016). The role of myokines in muscle health and disease. Curr. Opin. Rheumatol. 28, 661-666. doi: 10.1097/BOR.0000000000000337

Marklund, P., Mattsson, C. M., Wahlin-Larsson, B., Ponsot, E., Lindvall, B., Lindvall, L., et al. (2013). Extensive inflammatory cell infiltration in human skeletal muscle in response to an ultra-endurance exercise bout in experienced athletes. J. Appl. Physiol. 114, 66-72. doi: 10.1152/japplphysiol.01538.2011

Marqués-Jiménez, D., Calleja-González, J., Arratibel, I., Delextrat, A., and Terrados, N. (2016). Are compression garments effective for the recovery of exercise-induced muscle damage? A systematic review with meta-analysis. Physiol. Behav. 153, 133-148. doi: 10.1016/j.physbeh.2015.10.027

Methley, A. M., Campbell, S., Chew-Graham, C., McNally, R., and Cheraghi-Sohi, S. (2014). PICO, PICOS and SPIDER: a comparison study of specificity and sensitivity in three search tools for qualitative systematic reviews. BMC Health Serv. Res. 14:579. doi: 10.1186/s12913-014-0579-0

Moghadam-Kia, S., Oddis, C. V., and Aggarwal, R. (2016). Approach to asymptomatic creatine kinase elevation. Cleve. Clin. J. Med. 81, 37-42. doi: 10.3949/ccjm.83a.14120

Moldoveanu, A. I., Shephard, R. J., and Shek, P. N. (2001). The cytokine response to physical activity and training. Sport. Med. 31, 115-144. doi: 10.2165/00007256-200131020-00004

Mucci, P., Durand, F., Lebel, B., Bousquet, J., Préfaut, C., and Efaut, C. P. (1999). Interleukins 1-beta, -8 , and histamine increases in highly trained, exercising athletes. Med. Sci. Sport. Exerc. 32, 1094-1100. doi: 10.1097/00005768-200006000-00009

Nieman, D. C., Konrad, M., Henson, D. A., Kennerly, K., Shanely, R. A., and Wallner-Liebmann, S. J. (2012). Variance in the acute inflammatory response to prolonged cycling is linked to exercise intensity. J. Interf. Cytokine Res. 32, 12-17. doi: 10.1089/jir.2011.0038

Oishi, Y., and Manabe, I. (2018). Macrophages in inflammation, repair and regeneration. Int. Immunol. 30, 511-528. doi: 10.1093/intimm/dxy054

Ostrowski, K., Rohde, T., Asp, S., Schjerling, P., and Pedersen, B. K. (1999). Proand anti-inflammatory cytokine balance in strenuous exercise in humans. $J$. Physiol. 515, 287-291. doi: 10.1111/j.1469-7793.1999.287ad.x

Peake, J. M., Neubauer, O., Walsh, N. P., and Simpson, R. J. (2017). Recovery of the immune system after exercise. J. Appl. Physiol. 122, 1077-1087. doi: 10.1152/japplphysiol.00622.2016

Pedersen, B. K. (2017). Anti-inflammatory effects of exercise: role in diabetes and cardiovascular disease. Eur J Clin Invest. 47, 600-611. doi: 10.1111/eci.12781

Pescatello, L. S., Ross, A., Deborah, R., and D., T. P. (2013). "General principles of exercise prescription," in Guidelines for Exercise Testing and Presciption, 9th Edn. eds L. S. Pescatello, R. Arena, D. Riebe, and D. Paul (Philadelphia, PA: Thompson), 162-193.

Petersen, A. M. W., and Pedersen, B. K. (2005). The anti-inflammatory effect of exercise. J. Appl. Physiol. 98, 1154-1162. doi: 10.1152/japplphysiol.00164.2004

Roca-rodríguez, M. M., Garrido-sánchez, L., García-almeida, J. M., Ruiz-nava, J., Alcaide-torres, J., Gómez-gonzález, A., et al. (2015). Effects of exercise on inflammation in cardiac rehabilitation. Nutr. Hosp. 31, 2633-2640. doi: $10.3305 /$ nh.2015.31.6.8868

Santos, C. C., César, J., Neto, R., Bishop, N. C., and Lira, F. S. (2019). Interleukin 10 responses from acute exercise in healthy subjects: a systematic review. J. cell. Physiol. 234, 9956-9965. doi: 10.1002/jcp.27920

Schrödl, W., Büchler, R., Wendler, S., Reinhold, P., Muckova, P., Reindl, J., et al. (2016). Acute phase proteins as promising biomarkers: perspectives and limitations for human and veterinary medicine. Proteomics Clin. Appl. 10, 1077-1092. doi: 10.1002/prca.201600028

Schulz, K. F., and Atlman, D. G. M. D. (2010). CONSORT 2010 Statement: updated guidelines for repoting parallel group randomise trials. Ann. Int. Med. 152, 726-732. doi: 10.7326/0003-4819-152-11-201006010-00232

Shaw, D. M., Merien, F., Braakhuis, A., and Dulson, D. (2017). T-cells and their cytokine production: the anti-inflammatory and immunosuppressive effects of strenuous exercise. Cytokine 104, 136-142. doi: 10.1016/j.cyto.2017.10.001 
Silveira, L. S., Antunes Bde, M., Minari, A. L., dos Santos, R. V., Neto, JC., and Lira, F. S. (2016). Macrophage polarization: implications on metabolic diseases and the role of exercise. Crit. Rev. Eukaryot. Gene Expr. 26, 115-132. doi: 10.1615/CritRevEukaryotGeneExpr.2016015920

Spiropoulos, A., Goussetis, E., Margeli, A., Premetis, E., Skenderi, K., Graphakos, S., et al. (2010). Effect of inflammation induced by prolonged exercise on circulating erythroid progenitors and markers of erythropoiesis. Clin. Chem. Lab. Med. 48, 199-203. doi: 10.1515/CCLM.2010.034

Starkie, R., Ostrowski, S. R., Jauffred, S., Febbraio, M., and Pedersen, B. K. (2003). Exercise and IL-6 infusion inhibit endotoxin-induced TNF-alpha production in humans. FASEB J. 17, 884-886. doi: 10.1096/fj.02-0670fje

Stelzer, I., Kröpfl, J. M., Fuchs, R., Pekovits, K., Mangge, H., Raggam, R. B., et al. (2015). Ultra-endurance exercise induces stress and inflammation and affects circulating hematopoietic progenitor cell function. Scand. J. Med. Sci. Sport. 25, e442-450. doi: 10.1111/sms.12347

Suzuki, K., Nakaji, S., Yamada, M., Liu, Q., Kurakake, S., Okamura, N., et al. (2003). Impact of a competitive marathon race on systemic cytokine and neutrophil responses. Med. Sci. Sports Exerc. 35, 348-355. doi: 10.1249/01.MSS.0000048861.57899.04

Timmons, B. W., and Cieslak, T. (2008). Human natural killer cell subsets and acute exercise: a brief review. Exerc. Immunol. Rev. 14, 8-23.

Ulven, S. M., Foss, S. S., Skjolsvik, A. M., Stadheim, H. K., Myhrstad, M. C., Raael, E., et al. (2015). An acute bout of exercise modulate the inflammatory response in peripheral blood mononuclear cells in healthy young men. Arch. Physiol. Biochem. 121, 41-49. doi: 10.3109/13813455.2014. 1003566
Urrútia, G., and Bonfill, X. (2010). Declaración PRISMA: una propuesta para mejorar la publicación de revisiones sistemáticas y metaanálisis. Med. Clin. 135, 507-511. doi: 10.1016/j.medcli.2010.01.015

Viana, J. L., Kosmadakis, G. C., Watson, E. L., Bevington, A., Feehally, J., Bishop, N. C., et al. (2014). Evidence for anti-inflammatory effects of exercise in CKD. J. Am. Soc. Nephrol. 25, 2121-2130. doi: 10.1681/ASN.2013070702

von Elm, E., Altman, D., Egger, M., Pococ, S., Gotzsche, P., and Vandenbrouche, J. (2008). The strengthening of repoting of observational studies in epidemiology (STROBE)statement: guidelines for repoting observational studies. J. clin. Epidemiol. 61, 344-349. doi: 10.1016/j.jclinepi.2007.11.008

Wadley, A. J., Chen, Y.-W., Lip, G. Y. H., Fisher, J. P., and Aldred, S. (2015). Low volume-high intensity interval exercise elicits antioxidant and anti-inflammatory effects in humans. J. Sports Sci. 34, 1-9. doi: $10.1080 / 02640414.2015 .1035666$

Conflict of Interest: The authors declare that the research was conducted in the absence of any commercial or financial relationships that could be construed as a potential conflict of interest.

Copyright (C) 2020 Cerqueira, Marinho, Neiva and Lourenço. This is an open-access article distributed under the terms of the Creative Commons Attribution License (CC $B Y)$. The use, distribution or reproduction in other forums is permitted, provided the original author(s) and the copyright owner(s) are credited and that the original publication in this journal is cited, in accordance with accepted academic practice. No use, distribution or reproduction is permitted which does not comply with these terms. 